IDENTIFYING DYNAMICALLY YOUNG GALAXY GROUPS VIA WIDE-ANGLE TAIL GALAXIES: A CASE STUDY IN THE COSMOS FIELD AT $z=0.53$

This article has been downloaded from IOPscience. Please scroll down to see the full text article. 2010 ApJ 713484

(http://iopscience.iop.org/0004-637X/713/1/484)

The Table of Contents and more related content is available

Download details:

IP Address: 131.215.220.165

The article was downloaded on 09/04/2010 at 23:52

Please note that terms and conditions apply. 


\title{
IDENTIFYING DYNAMICALLY YOUNG GALAXY GROUPS VIA WIDE-ANGLE TAIL GALAXIES: A CASE STUDY IN THE COSMOS FIELD AT $z=0.53^{*}$
}

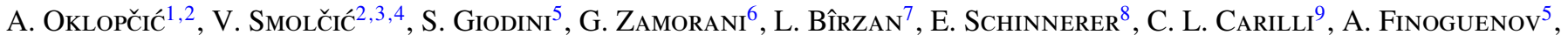 \\ S. Lilly ${ }^{10}$, A. KoeKemoer ${ }^{11}$, AND N. Z. ScOville ${ }^{2}$ \\ ${ }^{1}$ University of Zagreb, Physics Department, Bijenička cesta 32, 10002 Zagreb, Croatia \\ ${ }^{2}$ California Institute of Technology, MC 105-24, 1200 East California Boulevard, Pasadena, CA 91125, USA \\ ${ }^{3}$ ESO ALMA COFUND Fellow, European Southern Observatory, Karl-Schwarzschild-Strasse 2, 85748 Garching b. Muenchen, Germany \\ ${ }_{5}^{4}$ Argelander Institut for Astronomy, Auf dem Hügel 71, Bonn 53121, Germany \\ ${ }^{5}$ Max Planck Institut für Extraterrestrische Physik, D-85478 Garching, Germany \\ ${ }^{6}$ INAF-Osservatorio Astronomico di Bologna, via Ranzani 1, 40127 Bologna, Italy \\ ${ }^{7}$ Leiden Observatory, Leiden University, Oort Gebouw, P.O. Box 9513, 2300 RA Leiden, The Netherlands \\ ${ }^{8}$ Max Planck Institut für Astronomie, Königstuhl 17, Heidelberg D-69117, Germany \\ ${ }^{9}$ National Radio Astronomy Observatory, P.O. Box 0, Socorro, NM 87801-0387, USA \\ ${ }^{10}$ Eidgenössische Technische Hochschule - Zürich, CH-8093 Zürich, Switzerland \\ ${ }^{11}$ Space Telescope Science Institute, 3700 San Martin Drive, Baltimore, MD 21218, USA \\ Received 2009 November 6; accepted 2010 February 23; published 2010 March 23
}

\begin{abstract}
We present an analysis of a wide-angle tail (WAT) radio galaxy located in a galaxy group in the COSMOS field at a redshift of $z=0.53$ (hereafter CWAT-02). We find that the host galaxy of CWAT-02 is the brightest galaxy in the group although it does not coincide with the center of mass of the system. Estimating (1) the velocity of CWAT-02, relative to the intra-cluster medium, and (2) the line-of-sight peculiar velocity of CWAT-02's host galaxy, relative to the average velocity of the group, we find that both values are higher than those expected for a dominant galaxy in a relaxed system. This suggests that CWAT-02's host group is dynamically young and likely in the process of an ongoing group merger. Our results are consistent with previous findings showing that the presence of a WAT galaxy in a galaxy group or cluster can be used as an indicator of dynamically young non-relaxed systems. Taking the unrelaxed state of CWAT-02's host group into account, we discuss the impact of radio-active galactic nucleus (AGN) heating from CWAT-02 onto its environment, in the context of the missing baryon problem in galaxy groups. Our analysis strengthens recent results suggesting that radio-AGN heating may be powerful enough to expel baryons from galaxy groups.
\end{abstract}

Key words: cosmology: observations - galaxies: active - galaxies: evolution - galaxies: fundamental parameters radio continuum: galaxies

Online-only material: color figures

\section{INTRODUCTION}

Wide-angle tail (WAT) radio galaxies are radio galaxies whose jets are bent forming a wide $\mathrm{C}$ shape. They are usually found in dense environments, such as galaxy clusters and groups. It is believed that the characteristic morphology of WATs is a result of ram pressure being exerted on the radio jets, due to the relative motion of the host galaxy with respect to the intra-cluster medium (ICM; e.g., Begelman et al. 1979). WATs are generally associated with brightest cluster galaxies (Owen \& Rudnick 1976) and they are found to move with peculiar velocities of a few hundreds $\mathrm{km} \mathrm{s}^{-1}$ relative to the cluster center. Often, such velocities cannot explain the shape of the radio jets (e.g., Eilek et al. 1984). Therefore, an invoked scenario that can explain the bending of the jets is one in which the ICM gas has streaming flows driven by cluster/subcluster mergers. As a consequence, the ICM gas may have an associated bulk velocity relative with the potential and the galaxies (Klamer et al. 2004).

This picture is supported by observations which report the presence of WAT galaxies in connection with other indicators of a recent cluster merger, such as X-ray substructure (Burns et al. 1994), the elongation of the X-ray emission along the line that bisects the WAT (Gomez et al. 1997), and a significant offset

\footnotetext{
* Based on observations with the National Radio Astronomy Observatory, which is a facility of the National Science Foundation operated under cooperative agreement by Associated Universities, Inc.
}

$(\sim 100 \mathrm{kpc})$ of the WAT from the X-ray centroid (Sakelliou \& Merrifield 2000). These findings suggest that WATs are efficient tracers of dynamically young clusters (see, e.g., Smolčić et al. 2007, S07 hereafter). This feature, together with the ability to detect powerful radio galaxies with short exposure at high redshift, where dimming affects the optical and X-ray emission, makes WATs unique probes of clustering in the high-redshift universe (Blanton et al. 2003).

The interest in radio galaxies has lately been renewed because of the major role their active galactic nucleus (AGN) feedback may play in massive galaxy formation and evolution (Croton et al. 2006; Bower et al. 2006; Best et al. 2006; Smolčić et al. 2008), as well as heating of the gas in galaxy clusters/groups. In terms of the latter, radio galaxies have been proposed to solve the "cooling flow problem" in galaxy groups and clusters (see Fabian 1994 for a review) and explain the lack of baryons in galaxy groups (Bower et al. 2008; Short \& Thomas 2009; Giodini et al. 2009, 2010).

In recent cosmological simulations, gas is removed from within the cluster as a consequence of the mechanical heating by radio outflows of the central AGN. This scenario successfully reproduces the observed X-ray luminosity-temperature $\left(L_{X}-T\right)$ relation and the halo gas fractions (Bower et al. 2008; Short \& Thomas 2009). Only recently, this idea has been observationally supported by Giodini et al. (2010). Comparing the mechanical output energy of radio AGN, in a sample of COSMOS galaxy groups, with the group's binding energy, they show that the 
mechanical removal of intra-cluster gas in galaxy groups by radio-AGN heating may be energetically feasible. In particular, if WATs are efficient proxies for dynamically young, i.e., merging group environments, they then shed light onto the recent assembly history of the system. This is important in terms of the missing baryon problem on group scales as the binding energy in the final system is higher than that in the merging constituents, while the average power of radio-AGN outflows is expected to remain comparable (Smolčić et al. 2008). This highlights the importance of studying in detail the properties of peculiar radio galaxies within galaxy groups and their interaction with the surrounding environment.

Here, we present a multiwavelength study of a WAT galaxy at $z=0.53$ in the COSMOS field (CWAT-02 hereafter) and its environment. This system is particularly interesting as it is at intermediate redshift. Furthermore, CWAT-02 is a radio galaxy with a one-sided jet, and its host group has already been studied by Giodini et al. $(2009,2010)$ in the context of the missing baryon problem on galaxy group scales.

We report magnitudes in the $\mathrm{AB}$ system, adopt $H_{0}=$ $70, \Omega_{M}=0.3, \Omega_{\Lambda}=0.7$, and define the radio synchrotron spectrum as $F_{\nu} \propto v^{-\alpha}$, where $F_{\nu}$ is the flux density at frequency $\nu$ and $\alpha$ is the spectral index.

\section{DATA}

The data used in this paper have been obtained from the panchromatic (X-ray to radio) COSMOS $\square^{\circ}$ survey (Scoville et al. 2007a), the largest mosaic obtained to date with the Advanced Camera for Surveys on Hubble Space Telescope (HST; Koekemoer et al. 2007). In the UV to IR wavelengths, the field has been observed in $\sim 30$ (broad and narrow) photometric bands (with GALEX, Subaru, CFHT, UKIRT, and Spitzer; see Capak et al. 2007 for details). The large number of bands allows a very accurate determination of photometric redshifts (Ilbert et al. 2009; Salvato et al. 2009) yielding a dispersion $\left(\sigma_{\delta z /\left(1+z_{\text {spec }}\right)}\right.$; where $\left.\delta z=z_{\text {spec }}-z_{\text {phot }}\right)$ of $0.7 \%$ at $i_{A B}^{+}<22.5$, and $3.34 \%$ at $i_{A B}^{+}>22.5$. The spectroscopic data used for this work are drawn from the Sloan Digital Sky Survey (SDSS; York et al. 2000) and zCOSMOS (Lilly et al. 2007, 2009) surveys.

Within the VLA-COSMOS large project (Schinnerer et al. 2007), the full $2 \square^{\circ}$ field was observed at $1.4 \mathrm{GHz}$ with the NRAO Very Large Array (VLA) in A and C configurations. Additional A-array observations have been obtained for the inner $1 \square^{\circ}$ (Schinnerer et al. 2010) yielding a final rms of $\sim 8 \mu \mathrm{Jy}$ beam ${ }^{-1}$ in the central part of the field at a resolution of $1^{\prime \prime} .5 \times 1^{\prime \prime} .4$. The rms in the area around CWAT-02 is $10.5 \mu \mathrm{Jy}_{\text {beam }}{ }^{-1}$.

The COSMOS field has been observed at $324 \mathrm{MHz}(90$ $\mathrm{cm}$ ) with the VLA in A-array (2008 November) for a total of $24 \mathrm{hr}$ (for a detailed description, see V. Smolčić et al. 2010, in preparation). The full $2 \square^{\circ}$ field has been covered within one single pointing. The final map, used here, has been generated using the AIPS task IMAGR and a weighting scheme intermediate between natural and uniform (i.e., ROBUST $=0$ ). The reached $\mathrm{rms}$ (fairly uniform over the full $2 \square^{\circ}$ ) and resolution are $\sim 0.5 \mathrm{mJy} \mathrm{beam}^{-1}$ and $6^{\prime \prime} .4 \times 5^{\prime \prime} .4$, respectively. The rms in the area of CWAT-02 is $436 \mu \mathrm{Jy}_{\text {beam }}{ }^{-1}$.

X-ray observations of the COSMOS field have been performed with both XMM-Newton ( $1.5 \mathrm{Ms}$ covering $2 \square^{\circ}$; Hasinger et al. 2007) and Chandra (1.8 Ms in the inner $1 \square^{\circ}$; Elvis et al. 2009). Here, we make use of the galaxy cluster catalog described in detail in Finoguenov et al. (2007). Extended source detection was based on a wavelet analysis technique performed on the composite XMM-Newton and Chandra mosaic, after background and point-source subtraction. Each X-ray cluster candidate was further independently verified via an optical galaxy cluster search algorithm (making use of both the COSMOS photometric and spectroscopic redshifts). The final catalog contains $\sim 200$ galaxy clusters; the host group of CWAT-02 is identified as group 35, and its IAU designation is CXGG100049+0149.3.

\section{PROPERTIES OF THE WIDE-ANGLE TAIL GALAXY CWAT-02}

\subsection{Optical/IR}

The host galaxy of CWAT-02 is located at R.A. = $150.2066(10: 00: 49.59)$ and decl. $=1.8233(+01: 49:$ 23.85). It is a bright $\left(M_{I}=-23.04\right)$ spheroid-like red (rest frame $U-B=0.96$ ) galaxy with a spectroscopic (SDSS DR7; Abazajian et al. 2009) redshift of $z=0.5302 \pm 0.0002$. The stellar mass of the galaxy, as estimated by Smolčić et al. (2008) by fitting the global (from $3500 \AA$ to $2.5 \mu \mathrm{m}$ ) spectral energy distribution with the Bruzual \& Charlot (2003) stellar population synthesis models and a Chabrier (2003) initial mass function (IMF), is $3.5 \times 10^{11} M_{\odot}$. This is consistent with the highest mass galaxies known, which are often found in dense environments and usually associated with central brightest group galaxies (e.g., Best et al. 2005; von der Linden et al. 2007). The host galaxy has not been detected with Spitzer/MIPS at $24,70,160 \mu \mathrm{m}$, suggesting a low amount of dust and insignificant star formation. These properties are consistent with those of red-sequence passive galaxies.

\subsection{Radio}

\subsubsection{The Structure and Luminosity of CWAT-02}

The radio morphology of CWAT-02, shown in Figure 1, is C-shaped, consistent with the morphology of WAT galaxies. The central radio peak coincides with the above described elliptical galaxy at $z=0.5302 \pm 0.0002$. From the central radio core, a very luminous jet extends in the southwest direction out to a distance of $\sim 20^{\prime \prime}(\sim 120 \mathrm{kpc}$ at $z=0.53)$. It ends in a lobe that extends another $12^{\prime \prime}(\sim 80 \mathrm{kpc})$ in NW-SE direction (in the plane of the sky). The counterjet is not detected within the VLA-COSMOS sensitivity, however, the lobe $\sim 25^{\prime \prime}$ $(\sim 155 \mathrm{kpc}$ at $z=0.53)$ away from the central core is clearly visible. Prominent hot-spots are associated with both lobes. The asymmetry of the jet/counterjet luminosities that likely arises from Doppler boosting suggests a small inclination of the jets to the line of sight (LOS; see the following section). We estimate the direction of motion of CWAT-02 (relative to the ICM) toward SE (in the plane of the sky) by bisecting the bending angle $\left(\sim 115^{\circ}\right)$ formed by the lobes (see Figure 1).

In Figure 1, we also show the $324 \mathrm{MHz}$ map of CWAT-02. Due to the factor of $\sim 4$ lower resolution and significantly decreased sensitivity $\left(\sigma=0.436 \mathrm{mJy}\right.$ beam $\left.^{-1}\right)$, compared to the $1.4 \mathrm{GHz}$ data, it does not reveal as much structural detail as seen at 20 $\mathrm{cm}$. Nevertheless, the main features, such as the central core and lobes, are still discernible.

We use the $1.4 \mathrm{GHz}$ and $324 \mathrm{MHz}$ data to perform a spectral index analysis. We find that the region where the central core of CWAT-02 is located is characterized by a flat spectrum $(\alpha \lesssim 0.3)$ likely resulting from the multicomponent emission of the core. The spectral index steepens to $\alpha \sim 1$ in the area from the core toward the lobes. In the lobes the spectral index flattens again, suggesting possible particle re-acceleration in the hot-spots. The 

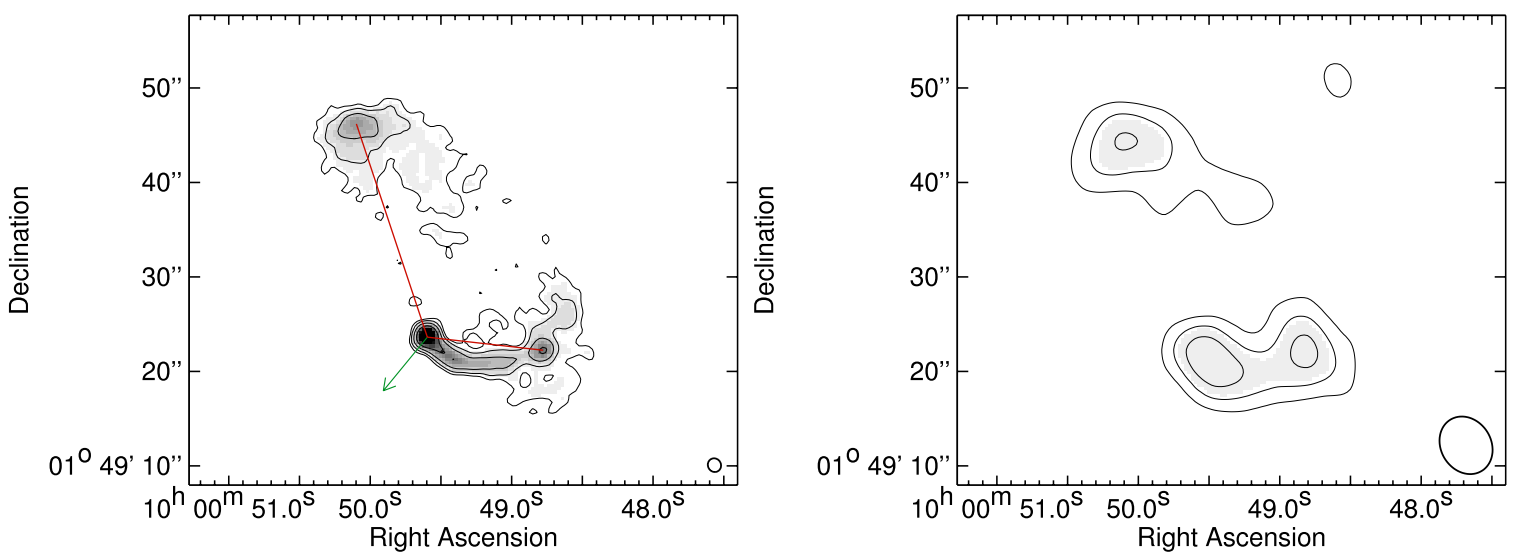

Figure 1. Left panel: $1.4 \mathrm{GHz}$ radio image of CWAT-02. The contour levels are at $2^{n} \cdot \sigma$, where $n=2,3,4 \ldots$, and $\sigma=10.5 \mu \mathrm{Jy}_{\text {beam }}{ }^{-1}$. The clean beam $\left(1^{\prime \prime} .5 \times 1^{\prime \prime} .4\right)$ is shown in the lower right corner. The arrow indicates the direction of the movement of CWAT-02 (relative to the ICM), in the plane of the sky. The lines connect the central radio core with the centers of the lobes, thus forming the bending angle of $\sim 115^{\circ}$. Right panel: $324 \mathrm{MHz}$ radio map of CWAT-02 with contours overlaid. The contour levels are in steps of $2 \sigma$, starting at $3 \sigma\left(\sigma=0.436 \mathrm{mJy}^{-1}\right.$ beam $\left.^{-1}\right)$. The resolution is $6^{\prime \prime} \cdot 44 \times 5^{\prime \prime} \cdot 38$, and the clean beam is shown in the lower right corner.

mean spectral index of the total source is $\alpha=0.6$, which we adopt for further calculations.

With a total flux density of $15.1 \mathrm{mJy}$ at $1.4 \mathrm{GHz}$ (see Schinnerer et al. 2010), and $18.2 \mathrm{mJy}$ at $324 \mathrm{MHz}$ (V. Smolčić et al. 2010, in preparation), the observed (not deboosted) restframe $1.4 \mathrm{GHz}$ and $324 \mathrm{MHz}$ luminosities of CWAT-02 are $1.4 \times$ $10^{25} \mathrm{~W} \mathrm{~Hz}^{-1}$ and $1.7 \times 10^{25} \mathrm{~W} \mathrm{~Hz}^{-1}$, respectively. The total radio luminosity, obtained by integrating the synchrotron spectrum (see, e.g., Equation (1) in S07) from $10 \mathrm{MHz}$ to 100 $\mathrm{GHz}$ is then $L_{\text {tot }}=2.7 \times 10^{42} \mathrm{erg} \mathrm{s}^{-1}$.

\subsection{Doppler Boosting and the Jet Velocity}

The radio jet luminosity of CWAT- 02 is highly asymmetric. A plausible explanation lies in Doppler boosting which causes the luminosity of the jet moving toward the observer to be amplified and the luminosity of the jet moving away to be suppressed, assuming the bulk velocity of the jet is relativistic.

For a jet moving at a speed of $v=\beta c$, the ratio of the rest-frame (Doppler boosted) luminosity $L(v)$ to the intrinsic luminosity $L_{0}(v)$ is given by (see, e.g., Lind \& Blandford 1985)

$$
\frac{L(\nu)}{L_{0}(\nu)}=[\gamma(1-\beta \cos \theta)]^{-(2+\alpha)} \equiv \delta^{2+\alpha},
$$

where $\gamma=\left(1-\beta^{2}\right)^{-1 / 2}$ is the Lorentz factor, $\theta$ is the jet orientation angle with respect to the LOS, and $\alpha$ is the spectral index $\left(F_{v} \propto v^{-\alpha}\right)$. The quantity

$$
\delta=[\gamma(1-\beta \cos \theta)]^{-1}
$$

is the so-called Doppler factor.

Applying Equation (1) to both the approaching and the receding jet and assuming that the intrinsic rest-frame luminosities of the two jets are the same, the ratio of jet to counterjet fluxes (or monochromatic luminosities) is obtained as

$$
R=\frac{L_{\mathrm{app}}}{L_{\mathrm{rec}}}=\frac{S_{\mathrm{app}}}{S_{\mathrm{rec}}}=\left(\frac{1+\beta \cos \theta}{1-\beta \cos \theta}\right)^{2+\alpha} .
$$

The flux of the southern jet of CWAT-02, assumed to be approaching out to the bending point, is $848 \mu \mathrm{Jy}$. For the northern jet, we can only place an upper limit on the flux. Taking a $3 \sigma$ value times the estimate of the area over which the jet extends, we obtain the flux of the northern jet to be

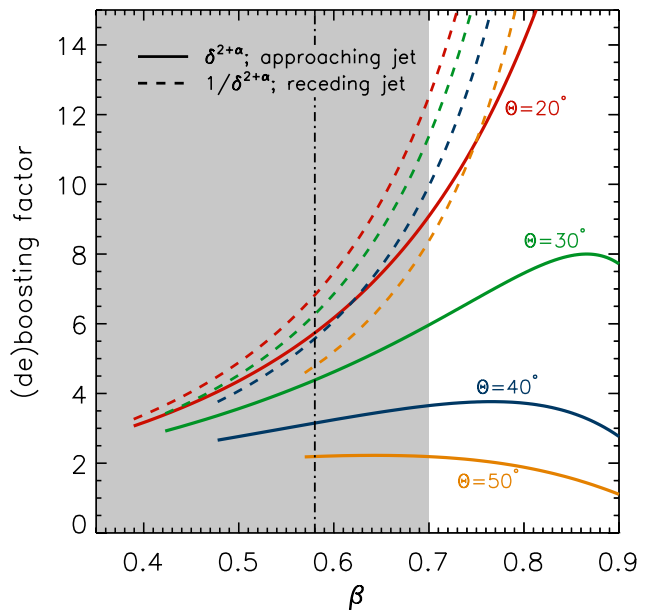

Figure 2. Doppler boosting factor of the approaching jet (solid lines) and the deboosting factor of the receding jet (dashed lines) as function of the bulk jet speed $\beta=v / c$ and the jet orientation angle to the line of sight $\theta$ for a range of jet-to-counterjet ratios $\geqslant 10$. The value of the speed of sound in a relativistic plasma $(0.58 c)$ is indicated by the dash-dotted vertical line. The typical jet speed range $(0.3 c-0.7 c$; adopted from Jetha et al. 2006) is designated by the gray-scale area. The sound speed sets a lower limit to CWAT-02 jet velocity (see the text for details).

$\lesssim 84 \mu \mathrm{Jy}$, yielding $R \gtrsim 10$. With $\alpha=1$ and $R \gtrsim 10$, Equation (2) yields $\beta \cos \theta \gtrsim 0.37$.

As only the lower limit on the jet flux ratios is known, and there is no independent constraint on either the jet velocity or the orientation angle, it is difficult to break the degeneracy between $\beta$ and $\theta$. However, we can place limits on the most likely velocity (and then on $\beta$ ) as follows. Figure 2 shows the Doppler boosting factor of the approaching jet $\left(L_{\mathrm{app}} / L_{0}\right.$; solid lines) and the deboosting factor of the receding jet $\left(L_{0} / L_{\mathrm{rec}}\right.$; dashed lines) as a function of the speed of the jet and the inclination angle, assuming a range of flux ratios $R \gtrsim 10$. For each $\theta$, assuming the same $\beta$ for the two jets, the expected $R$ is $\sim 10$ for a beta value corresponding to the left limit of the continuous lines (boosting for the approaching jet) and rapidly increases for higher $\beta$. We can constrain a possible range of $\beta$ from the observations. The first constraint on the jet speed arises from the hot-spots in CWAT-02. Assuming the hot-spots are jet-termination shocks (as in FR II sources), the presence of a hot-spot indicates that the speed of the jet is higher then the internal speed of sound $(\approx 0.58 c$ for a relativistic plasma; see, e.g., Jetha et al. 2006). 

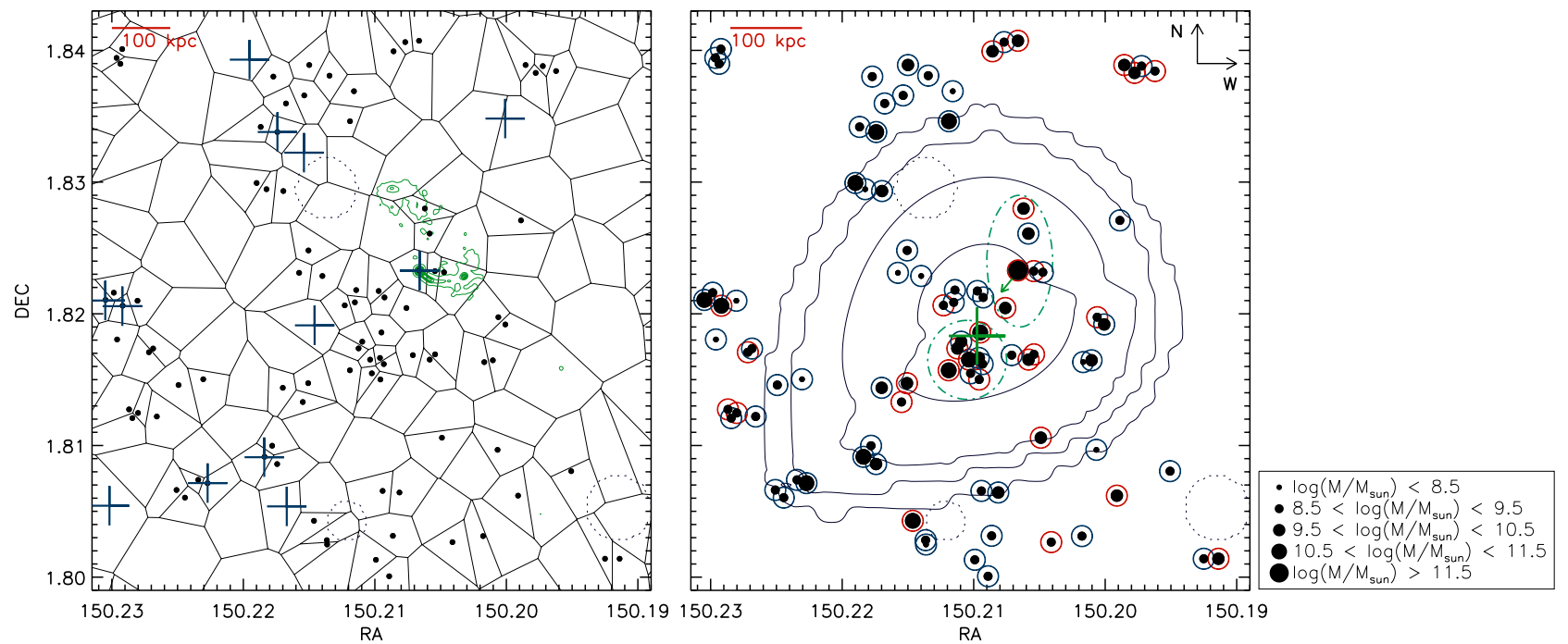

Figure 3. Left panel: voronoi tessellation of the area around CWAT- $02\left(2^{\prime} \cdot 64 \times 22^{\prime} \cdot 64\right.$, corresponding to $\sim 1 \mathrm{Mpc} \times 1 \mathrm{Mpc}$, which is $\sim 1 \%$ of the total area analyzed) overlaid with radio contours. Filled dots represent "high density" galaxies that are less than $\sim 500 \mathrm{kpc}$ away from CWAT-02. Crosses indicate the position of the galaxies for which a spectroscopic redshift is available. Masked-out regions in the optical, around saturated objects, are marked with dotted circles. The right panel shows the same area as in the left panel, but overlaid with X-ray contours. The galaxies are represented by encircled dots, where the size of each dot is related to the stellar mass of the corresponding galaxy, as indicated in the legend. The color of the circle matches the color of the galaxy, retrieved from the color-magnitude diagram (see Figure 4). The arrow indicates the velocity direction of CWAT-02 (projected onto the plane of the sky; see Figure 1). The cross marks the center of mass of the galaxies within the X-ray emission region (see the text for details). Dash-dotted lines indicate the two assumed merging galaxy subgroups, one is associated with the center of mass of the system, and the other contains CWAT- 02 .

(A color version of this figure is available in the online journal.)

This then sets a likely lower limit on CWAT-02's jet speed of $\sim 0.6 c$. Secondly, based on a sample of 30 WATs, combined with Monte Carlo simulations to infer their bulk jet velocities, Jetha et al. (2006) found typical WAT jet speeds to be in the range $0.3 c-0.7 c$ (indicated by the gray-shaded area in Figure 2). Thus, combining the above two arguments suggests that a likely bulk velocity of CWAT-02's jets is somewhere in the range $0.6 c-0.7 c$. Depending on the angle between the jets and the LOS, the observed luminosity of the approaching jet can then be boosted by a factor ranging from $\sim 2$ (for $\theta \sim 50^{\circ}$ ) to $\sim 9$ (for $\theta \sim 20^{\circ}$ ), while the luminosity of the receding jet would be deboosted by factors $\sim 5$ and $\sim 13$ for the same $\theta$ values (see Figure 2). Taking the values for the maximum (de)boosting $\left(\theta=20^{\circ} ; \beta=0.7\right)$ and assuming that the intrinsic (non-boosted) luminosities of the jets are equal, we find that the observed total jet flux (the sum of the approaching and receding jet flux densities) is a factor of $\sim 4.5$ higher than the intrinsic one. This suggests that the total intrinsic luminosity of the jets is likely less than a factor of 5 lower compared to the observed value. However, the observed jet flux $(1.77 \mathrm{mJy}$ at $20 \mathrm{~cm})$ accounts for only $12 \%$ of the total observed flux of CWAT-02 (15.1 mJy). Thus, the remaining $88 \%$ arises from non-boosted features, such as the core and the lobes. Therefore, Doppler boosting affects only a minor portion of the total flux of the source, and the observed value of the luminosity, reported in Section 3.2., is a good estimate of the total intrinsic luminosity.

\section{THE ENVIRONMENT OF CWAT-02}

\subsection{Voronoi Tessellation-based Approach: the CWAT-02 Host Group}

To identify the galaxy overdensity associated with CWAT02, we use the Voronoi tessellation-based approach (VTA; e.g., S07; Ramella et al. 2001; Kim et al. 2002), a method particularly favorable for revealing substructure in overdense environments. A Voronoi tessellation on a two-dimensional plane containing positions of galaxies within a fixed redshift range, divides the plane into convex polygons where each polygon contains one galaxy. The inverse of the area of each polygon corresponds then to the effective local density of the galaxy.

To determine the clustering in the CWAT-02 region, we select galaxies that are less than $\sim 13^{\prime}(5 \mathrm{Mpc}$ at $z=0.53$ ) away from CWAT-02, and have photometric redshifts within $\Delta z=3 \sigma_{\delta z /\left(1+z_{s}\right)} \cdot(1+0.53)$, where $\sigma_{\delta z /\left(1+z_{s}\right.}$ is the typical photometric redshift error (see Section 1). To estimate the background density, we randomly distribute the same number of galaxies $(11,162)$ over the same area 100 times. We then apply the VTA to each generated field (see Botzler 2004), and calculate the mean local density $\overline{\rho_{b k g}}$ and its standard deviation $\sigma_{b k g}$ (see S07 for more details). All the Voronoi tiles with densities exceeding the value of $\overline{\rho_{b k g}}+10 \sigma_{b k g}$ are considered overdense.

The spatial distribution of these "overdense" galaxies, shown in Figure 3, in the immediate surrounding of CWAT-02 shows an elongation in the NW-SE direction, with two discernible high-mass peaks indicated in the right panel of Figure 3. There is a strong accumulation of very bright, red, and massive earlytype galaxies close to the center of the X-ray emission (see below). However, the most massive and brightest galaxy is CWAT-02's host galaxy. With a few other galaxies, CWAT-02 makes the northwestern elongation of the central massive galaxy accumulation.

In Figure 4, we show the color-magnitude diagram of the "high-density" galaxies (shown in Figure 3). The red sequence is clearly visible. It is interesting that although CWAT-02's host galaxy is the brightest one in the system, the next three brightest red-sequence galaxies are all concentrated in the clump close to the center of the X-ray emission, offset from CWAT-02's host by $\sim 19^{\prime \prime}$, i.e., $\sim 120 \mathrm{kpc}$ at $z=0.53$. This suggests an unrelaxed state of the cluster and will be further discussed in Section 5 .

The existence of a galaxy agglomeration around CWAT-02 is verified by 12 spectroscopic redshifts $(z$ in the range from 


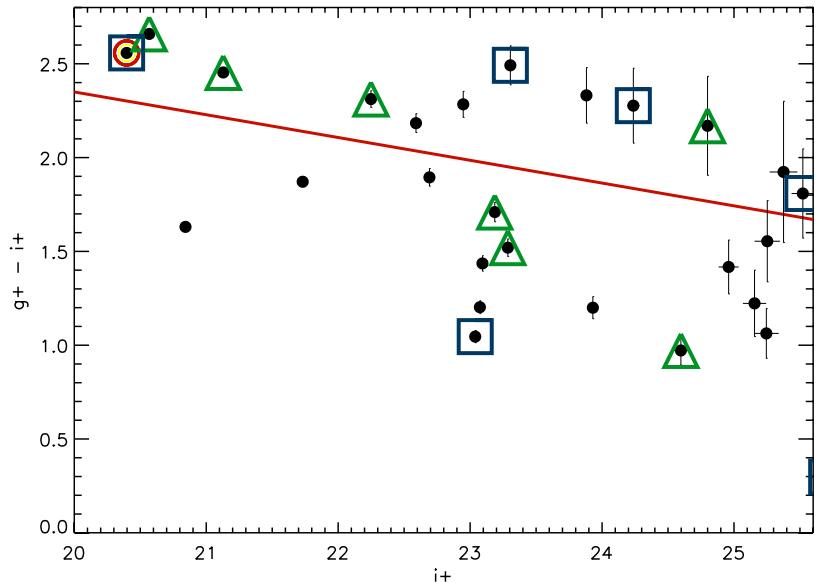

Figure 4. Color-magnitude diagram of the galaxies around CWAT-02. We use the indicated line to separate blue from red galaxies. A distinct red sequence is discernible. The triangles indicate the members of the subgroup located near the center of the X-ray emission, and the squares mark the galaxies forming the subgroup dominated by CWAT-02 (see Figure 3). CWAT-02 is indicated by the encircled dot.

(A color version of this figure is available in the online journal.)

0.5242 to 0.5341 , with an average redshift of 0.5291 ) present in the region of interest (see Figure 3 and Table 1). Furthermore, the entire structure is embedded in the densest large-scale structure (LSS) component at $z=0.53$ (Scoville et al. 2007b). It is located at the northwestern outskirts of this LSS component which is fairly elongated, and extends over $\sim 5^{\prime}(\sim 2 \mathrm{Mpc}$ at $z=0.53)$ in the NW-SE direction.

\subsection{X-ray Properties of the Galaxy Group}

The extended X-ray emission from the diffuse hot gas within the galaxy group hosting CWAT-02 is shown in Figure 3 (right panel). As reported in the COSMOS galaxy cluster catalog (Finoguenov et al. 2007), the total X-ray luminosity of the ICM, in the $0.1-2.4 \mathrm{keV}$ band, is $L_{X}=$ $(6 \pm 1) \times 10^{42} \mathrm{erg} \mathrm{s}^{-1}$, the total mass embedded within $r_{200}$ is $(5.2 \pm 0.4) \times 10^{13} M_{\odot}$, and the temperature is $k T=1.04 \pm$ $0.06 \mathrm{keV}$, thus consistent with properties of a galaxy group. The total mass and temperature have been estimated using the scaling relations from Leauthaud et al. (2010). The group's extended Xray emission has been identified at high significance. We note that several strong X-ray point sources have been subtracted from the group's emission (NW and SE from CWAT-02), thus potentially biasing the X-ray group center determination. To constrain the group center more robustly, we compute the center of mass using the potential group member galaxies within the region of X-ray emission (see Section 4.1). The center of mass (see Figure 3 ) roughly coincides with the agglomeration of red, massive galaxies SE of CWAT-02, and is very close to the peak of the X-ray emission.

In addition to the group's extended X-ray emission, CWAT-02 itself is detected as a faint source in the Chandra data (identified as Chandra source 1292; F. Civiano et al. 2010, in preparation), with a flux of $4.5 \times 10^{-16} \mathrm{erg} \mathrm{cm}^{-2} \mathrm{~s}^{-1}$ in the soft band $(0.5-2.0$ $\mathrm{keV}$; Elvis et al. 2009).

\section{DISCUSSION}

\subsection{WATs as Tracers of Dynamically Young Clusters}

The existence of CWAT-02's host galaxy group at $z=0.53$ has been independently determined in three different wavelength
Table 1

Spectroscopic Redshifts for the Galaxies Around CWAT-02, drawn from zCOSMOS (Lilly et al. 2007, 2009) and SDSS (Abazajian et al. 2009)

\begin{tabular}{cccc}
\hline \hline R.A. & Decl. & Origin & $z_{\text {spec }}$ \\
\hline 150.20009 & 1.83482 & zCOSMOS & 0.5296 \\
150.20661 & 1.82327 & SDSS & 0.5302 \\
150.21458 & 1.81912 & zCOSMOS & 0.5289 \\
150.21539 & 1.83223 & zCOSMOS & 0.5341 \\
150.21670 & 1.80537 & zCOSMOS & 0.5294 \\
150.21741 & 1.83380 & zCOSMOS & 0.5314 \\
150.21841 & 1.80911 & zCOSMOS & 0.5280 \\
150.21953 & 1.83930 & zCOSMOS & 0.5280 \\
150.22272 & 1.80714 & zCOSMOS & 0.5300 \\
150.22920 & 1.82058 & zCOSMOS & 0.5242 \\
150.23018 & 1.80542 & zCOSMOS & 0.5301 \\
150.23050 & 1.82101 & zCOSMOS & 0.5250 \\
\hline
\end{tabular}

windows (radio, optical, and X-ray). We find that although it is the brightest and most massive galaxy in the analyzed region, CWAT-02 is not, as one would expect in a relaxed system, at the center of its host group, but offset from it to the NW. The next three brightest massive galaxies are located in the agglomeration closest to the center of mass of the group. This is an indication of a disturbed system that is probably undergoing a process of group or sub-group merger. To test this idea in the following, we put constraints on the velocity of CWAT-02's host galaxy relative to the ICM that is needed to explain the observed bending of the radio jets, and compare it to velocities expected in relaxed systems.

If the bending of the radio jet is generated by ram pressure, then a non-negligible motion of the galaxy relative to the ICM must exist. To shed light on this, we make use of the model developed by Begelman et al. (1979), which assumes that the ram pressure exerted on the galaxy, as it moves through the ICM, is balanced by the centrifugal force exerted by the jet as the jet moves with a bulk velocity outwards from the host galaxy and thereby curves. We make use of the Euler equation, written in the form

$$
\frac{\rho_{\mathrm{ICM}} v_{\mathrm{gal} / \mathrm{ICM}}^{2}}{h}=\frac{\rho_{j} v_{j}^{2}}{R_{j}},
$$

where $v_{j}$ is the bulk jet speed, $h$ is the jet-scale height, $R_{j}$ is the radius of curvature of the jet, $v_{\mathrm{gal} / \mathrm{ICM}}$ is the galaxy velocity relative to the ICM, and $\rho_{\mathrm{ICM}}$ and $\rho_{j}$ are the ICM and jet densities, respectively. Although this model presumes a nonrelativistic jet velocity, it is a reasonable approximation in case of a light and moderately relativistic jet (see Hardcastle et al. 2005; Jetha et al. 2006). For CWAT-02, we estimate a scale height of $h \sim 2^{\prime \prime} .2(\sim 14 \mathrm{kpc}$ at $z=0.53)$ and a radius of curvature of $R_{j} \sim 99^{\prime \prime} 0(\sim 57 \mathrm{kpc}$ at $z=0.53)$. Note, however, that the jet and ICM densities for the CWAT-02 system are not well constrained. For this reason in Figure 5 we show the dependence of $v_{\text {gal } / \mathrm{ICM}}$ on the jet to ICM density ratio, $\rho_{j} / \rho_{\text {ICM }}$. In general, typical $\rho_{j} / \rho_{\text {ICM }}$ values used in hydrodynamical simulations of radio jets are of the order of $10^{-4}-10^{-2}$ (e.g., Rossi et al. 2004). Thus, for the lower limit value of $\rho_{j} / \rho_{\mathrm{ICM}}=10^{-4}$, we obtain a velocity of $v_{\mathrm{gal} / \mathrm{ICM}} \sim 900 \mathrm{~km} \mathrm{~s}^{-1}$. Note that even for density ratios lower than this value $v_{\text {gal } / \text { ICM }}$ stays significant, i.e., more than several hundred $\mathrm{km} \mathrm{s}^{-1}$.

Based on our spectroscopic data, we can further put limits on the peculiar LOS velocity of CWAT-02's host galaxy. Note, however, that this is based on the assumption that the galaxies in the core of the group (surrounding CWAT-02) have a similar 


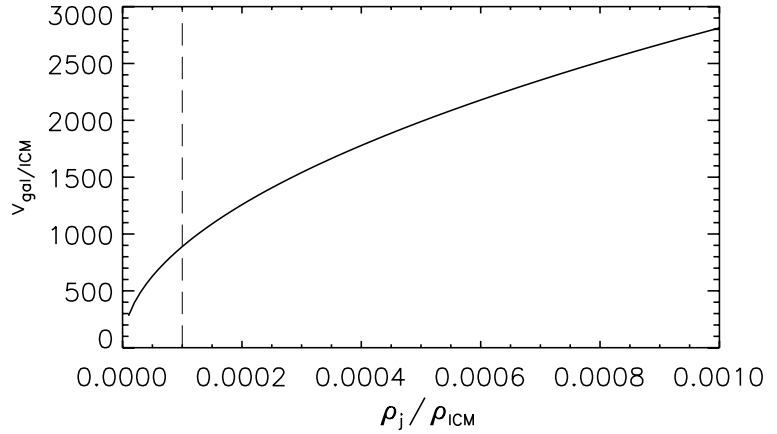

Figure 5. Velocity of CWAT-02's host galaxy relative to the ICM (estimated via Equation (3)) as a function of the jet to ICM density ratio, $\rho_{j} / \rho_{\text {ICM }}$ (thick line). The dashed line shows a low $\rho_{j} / \rho_{\text {ICM }}$ limit, typically assumed in hydrodynamical simulations (see the text for more details).

velocity distribution as those with spectroscopic redshifts that are located predominantly at the outskirts of the system. With this in mind, for the 12 galaxies from Table 1 we compute the LOS velocities as $v=z \cdot c$, where $v$ is the velocity, $z$ is the redshift, and $c$ is the speed of light. To estimate the median velocity and the velocity spread for CWAT-02's group, we use biweight statistics that was shown to be superior for samples containing $\sim 10$ galaxies (Beers et al. 1990). The redshift distribution is shown in Figure 6. The obtained biweight velocity dispersion is $437 \mathrm{~km} \mathrm{~s}^{-1}$. Note that based on the X-ray luminosity of the group, a velocity dispersion of $300-500 \mathrm{~km} \mathrm{~s}^{-1}$ is expected (Mulchaey 2000).

The velocity difference between CWAT-02's host galaxy and the biweight mean velocity of all the group galaxies is $244 \mathrm{~km} \mathrm{~s}^{-1}$. This is higher than the expected LOS peculiar velocities of $\lesssim 150 \mathrm{~km} \mathrm{~s}^{-1}$ of brightest group galaxies located in relaxed systems (Beers et al. 1995; see also Oegerle \& Hill 2001). Thus, it independently suggests a disturbed state of the system. The lower limit of the relative velocity between the CWAT-02's host galaxy and the ICM, in the projected plane of the sky, of $\sim 900 \mathrm{~km} \mathrm{~s}^{-1}$ is noticeably higher than the derived peculiar LOS velocity of the galaxy of $244 \mathrm{~km} \mathrm{~s}^{-1}$. Such a discrepancy suggests that a significant fraction of the relative velocity between the CWAT-02's host galaxy and the ICM likely arises from the bulk motion of the ICM itself. Large bulk velocities of the ICM can be caused by group mergers (Roettiger et al. 1996; Sato et al. 2008) that can also explain the elongation of the X-ray emission that is observed in CWAT-02's host group (Roettiger et al. 1996).

On large scales, the CWAT-02 host group is part of the strongest LSS component at $z=0.53$ (Scoville et al. 2007b), which extends over $2 \mathrm{Mpc}$ in the NW-SE direction, coincident with the elongation of the X-ray and optical distributions, as well as the motion of CWAT-02, relative to the ICM (projected on the plane of the sky). Given the location of the group at the NW outskirts of the LSS component, it is plausible to expect that the entire group will eventually migrate toward the center of the LSS component, and form a massive relaxed cluster.

Our results also show that WAT galaxies are efficient tracers of LSS overdensities. This is further strengthened by an analysis of the large-scale environment of $\sim 10$ other WATs in the COSMOS field (see Schinnerer et al. 2007) that yields that all (but one ${ }^{12}$ )

\footnotetext{
12 The galaxy has a C-shaped radio morphology. Its optical counterpart is a type $1 \mathrm{AGN}$ at $z=1.35$, and the radio jets are strongly Doppler boosted. Thus, the apparent bending of the jets may possibly arise from relativistic effects rather than the motion of the galaxy through a dense medium.
}

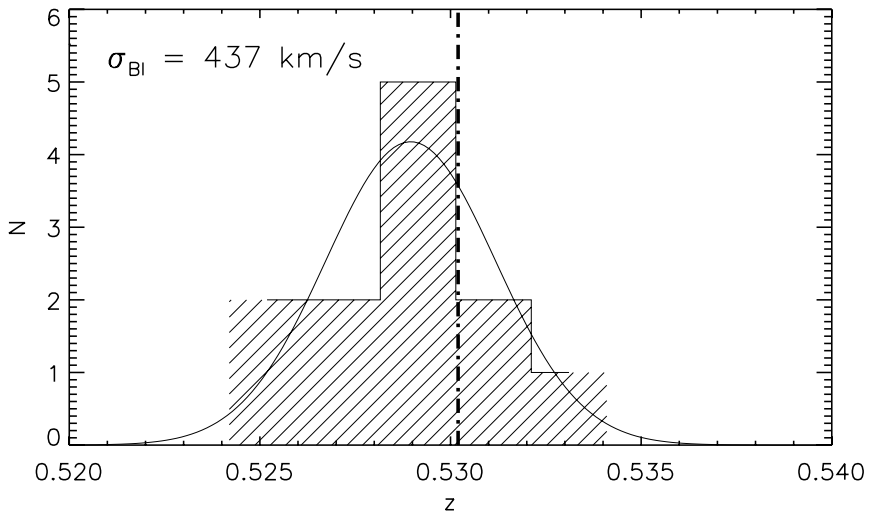

Figure 6. Redshift distribution of the 12 galaxies in the CWAT-02 region with spectroscopic redshifts (see Table 1; dashed histogram). Biweight statistics has been used to infer the spread $\sigma_{\mathrm{BI}}$ for the system (indicated in the panel). The dash-dotted line corresponds to the velocity of CWAT-02's host galaxy, offset from the mean velocity by $244 \mathrm{~km} \mathrm{~s}^{-1}$ (see the text for details).

are associated with LSS overdensity peaks, typically extending over $2 \mathrm{Mpc}$ (see also S07).

In summary, our results support the hypothesis that WAT galaxies inhabit gravitationally disturbed systems in the process of formation. This is consistent with previous results (e.g., Pinkney et al. 1994; Loken et al. 1995; Gomez et al. 1997; Sakelliou et al. 1996; Sakelliou \& Merrifield 2000) and demonstrates the usefulness of WATs as tracers of dynamically young groups and clusters. In the following analysis, we demonstrate the value of identifying merging systems, in the context of the missing baryon problem in galaxy groups.

\subsection{Can Radio-AGN Heating Expel Baryons from CWAT-02's Host Group?}

The interest in radio galaxies has been renewed in the last years as radio-AGN heating is regularly invoked in cosmological models as a key ingredient to explain the observed galaxy and galaxy cluster/group properties (Croton et al. 2006; Bower et al. 2006; Bower et al. 2008). Recent cosmological models assume that radio-AGN heating is powerful enough to expel a fraction of baryons from the cluster/group potential well (Bower et al. 2008). Such a process provides a satisfactory solution for the missing baryon problem on galaxy group scales, i.e., it can potentially reconcile the observed discrepancy between the baryon to dark matter mass ratio in galaxy groups and the WMAP-CMB value (see Giodini et al. 2009, 2010, and references therein). The first observational support for this scenario has been presented by Giodini et al. (2010). For each of the $16 \mathrm{X}$-ray-selected galaxy groups in the COSMOS field that host a radio galaxy, Giodini et al. have computed the output energy that the radio galaxy exerts onto its environment (over the host galaxy's lifetime), as well as the binding energy of the ICM. Comparing these two for all systems, they find that the energetics of radio AGN, computed in this way, may account for expelling gas from the group's potential well. One of the 16 systems analyzed by Giodini et al. (2010; identified as XID35) is CWAT-02's host group for which they infer a mechanical radio output energy of $(0.2-9.9) \times 10^{61} \mathrm{erg}$ (the range is a result of the 0.85 dex scatter in the scaling relations used to obtain this result; see Birzan et al. 2008). They find a comparable X-ray binding energy of (2.4-4) $\times 10^{61} \mathrm{erg}$, suggesting that the radioAGN heating done by CWAT-02 is powerful enough to expel gas from its host group. We discuss below how the findings based 
on the in-depth analysis of the CWAT-02 host system presented here affect this result.

Our Doppler boosting analysis, presented in Section 3.3, has shown that the relativistic boosting does not significantly affect the total intrinsic radio luminosity of CWAT-02, which was used to compute the output energy by Giodini et al. (2010). Therefore, Doppler boosting of the jets of CWAT-02 does not substantially alter the above given radio output energy. However, it is possible that massive galaxy members in CWAT-02's host group, that are currently radio-silent, have experienced radioAGN phases in their past and thereby contributed to the energy budget required to expel baryons from the group. In general, radio-emission in central massive galaxies located in galaxy clusters or groups is thought to originate from a self-regulating process (Best et al. 2005; Hardcastle et al. 2007; Merloni \& Heinz 2008; Smolčić et al. 2009). In this picture, radio activity is induced via cooling of hot gas onto the galaxy (Hardcastle et al. 2007; Merloni \& Heinz 2008). In turn, radio outflows heat the surrounding gas thus limiting its inflow and terminating the source of radio emission. The brightest, reddest, and most massive galaxies in CWAT-02's host group are dispersed in two clumps (see Figure 3), which is likely a result of group merger (as discussed in the previous section). Hence, it is possible that (some of) the most prominent galaxies in the merging system were once the central galaxies in the merging constituents. As such, based on the above outlined scenario, in these galaxies reoccurring radio outflows (that couple with the ICM) would be expected (Fabian 1994; Birzan et al. 2004, 2008; Giodini et al. 2009 , 2010). While not much change in radio power of such massive galaxies is (on average) expected with cosmic time (see Smolčić et al. 2009), the binding energy of the merging constituents could have been substantially lower (compared to the merged system), thus facilitating the removal of gas. In summary, the radio output energy budget in CWAT-02's host group may be powerful enough to expel baryons from its host group, strengthening the results of Giodini et al. (2010) who showed that radio-AGN heating may (on average) account for the missing baryons in galaxy groups.

\section{SUMMARY}

We have presented an analysis of a WAT galaxy in the COSMOS field (CWAT-02) and its host galaxy group at $z=0.53$. The in-depth study of the galaxy's environment, morphology, and velocity suggests an unrelaxed state of the host group, possibly caused by a galaxy group merger. This is consistent with the idea that WAT galaxies can be used as good tracers of dynamically young, unrelaxed systems. The analysis of radio-energy outflows from CWAT-02 suggests that the outflows (over the host galaxy's lifetime) may be powerful enough to expel gas from the group.

A.O. thanks California Institute of Technology for generous support through NASA grants 1292462 and 1344606. V.S. acknowledges support from the Owens Valley Radio Observatory, which is supported by the National Science Foundation through grant AST-0838260. The research leading to these results has received funding from the European Community's Seventh Framework Programme (FP7/2007-2013) under grant agreement 229517. V.S. and A.O. thank Unity through Knowledge Fund (www.ukf.hr) for collaboration support through the "Homeland Visit" grant. S.G. acknowledges support by the DFG Cluster of Excellence "Origin and Structure of the Universe" (www.universe-cluster.de). C.C. acknowledges support from the
Max-Planck Society and the Alexander von Humboldt Foundation through the Max-Planck-Forschungspreis 2005. G.Z. acknowledges support from an INAF contract PRIN-2007/ 1.06.10.08 and an ASI grant ASI/COFIS/WP3110 I/026/ $07 / 0$.

\section{REFERENCES}

Abazajian, K. N., et al. 2009, ApJS, 182, 543

Beers, T. C., Flynn, K., \& Gebhardt, K. 1990, AJ, 100, 32

Beers, T. C., Kriessler, J. R., Bird, C. M., \& Huchra, J. P. 1995, AJ, 109, 874

Begelman, M. C., Rees, M. J., \& Blandford, R. D. 1979, Nature, 279, 770

Best, P. N., Kaiser, C. R., Heckman, T. M., \& Kauffmann, G. 2006, MNRAS, 368, L67

Best, P. N., et al. 2005, MNRAS, 362, 9

Birzan, L., McNamara, B. R., Nulsen, P. E. J., Carilli, C. L., \& Wise, M. W. 2008, ApJ, 686, 859

Birzan, L., Rafferty, D. A., McNamara, B. R., Wise, M. W., \& Nulsen, P. E. J. 2004, ApJ, 607, 800

Blanton, E. L., Gregg, M. D., Helfand, D. J., Becker, R. H., \& White, R. L. 2003, AJ, 125, 1635

Botzler, C. S. 2004, PhD thesis, Ludwig-Maximilians-Univ. München

Bower, R. G., McCarthy, I. G., \& Benson, A. J. 2008, MNRAS, 390 , I399

Bower, R. G., et al. 2006, MNRAS, 370, 645

Bruzual, G., \& Charlot, S. 2003, MNRAS, 344, 1000

Burns, J. O., Rhee, G., Owen, F. N., \& Pinkney, J. 1994, ApJ, 423, 94

Capak, P., et al. 2007, ApJS, 172, 99

Chabrier, G. 2003, PASP, 115, 763

Croton, D. J., et al. 2006, MNRAS, 365, 11

Eilek, J. A., Burns, J. O., O’Dea, C. P., \& Owen, F. N. 1984, ApJ, 278, 37

Elvis, M., et al. 2009, ApJS, 184, 158

Fabian, A. C. 1994, ARA\&A, 32, 277

Finoguenov, A., et al. 2007, ApJS, 172, 182

Giodini, S., et al. 2009, ApJ, 703, 982

Giodini, S., et al. 2010, arXiv:1002.4212

Gomez, P. L., Ledlow, M. J., Burns, J. O., Pinkney, J., \& Hill, J. M. 1997, AJ, 114,1711

Hardcastle, M. J., Evans, D. A., \& Croston, J. H. 2007, MNRAS, 376, 1849

Hardcastle, M. J., Sakelliou, I., \& Worrall, D. M. 2005, MNRAS, 359, 1007

Hasinger, G., et al. 2007, ApJS, 172, 29

Ilbert, O., et al. 2009, ApJ, 690, 1236

Jetha, N. N., Hardcastle, M. J., \& Sakelliou, I. 2006, MNRAS, 368, 609

Kim, D. C., Veilleux, S., \& Sanders, D. B. 2002, ApJS, 143, 277

Klamer, I., Subrahmanyan, R., \& Hunstead, R. W. 2004, MNRAS, 351, 101

Koekemoer, A. M., et al. 2007, ApJS, 172, 196

Leauthaud, A., et al. 2010, ApJ, in press (arXiv:0910.5219)

Lilly, S. J., et al. 2007, ApJS, 172, 70

Lilly, S. J., et al. 2009, ApJS, 184, 218

Lind, K. R., \& Blandford, R. D. 1985, ApJ, 295, 358

Loken, C., Roettiger, K., Burns, J. O., \& Norman, M. 1995, ApJ, 445, 80

Merloni, A., \& Heinz, S. 2008, MNRAS, 388, 1011

Mulchaey, J. S. 2000, ARA\&A, 38, 289

Oegerle, W. R., \& Hill, J. M. 2001, AJ, 122, 2858

Owen, F. N., \& Rudnick, L. 1976, ApJ, 205, L1

Pinkney, J., Burns, J. O., \& Hill, J. M. 1994, AJ, 108, 2031

Ramella, M., Boschin, W., Fadela, D., \& Nonino, M. 2001, A\&A, 368, 776

Roettiger, K., Burns, J. O., \& Loken, C. 1996, ApJ, 473, 651

Rossi, P., Bodo, G., Massaglia, S., Ferrari, A., \& Mignone, A. 2004, Ap\&SS, 293, 149

Sakelliou, I., \& Merrifield, M. R. 2000, MNRAS, 311, 649

Sakelliou, I., Merrifield, M. R., \& McHardy, I. M. 1996, MNRAS, 283, 673

Salvato, M., et al. 2009, ApJ, 690, 1250

Sato, K., Matsushita, K., Ishisaki, Y., Yamasaki, N. Y., Ishida, M., Sasaki, S., \& Ohashi, T. 2008, PASJ, 60, S333

Schinnerer, E., et al. 2007, ApJS, 172, 46

Schinnerer, E., et al. 2010, ApJS, submitted

Scoville, N., et al. 2007a, ApJS, 172, 1

Scoville, N., et al. 2007b, ApJS, 172, 150

Short, C. J., \& Thomas, P. A. 2009, ApJ, 704, 915

Smolčić, V., et al. 2007, ApJS, 172, 295

Smolčić, V., et al. 2008, ApJS, 177, 14

Smolčić, V., et al. 2009, ApJ, 696, 24

von der Linden, A., Best, P. N., Kauffmann, G., \& White, S. D. M. 2007, MNRAS, 379, 867

York, D. G., et al. 2000, AJ, 120, 1579 